\title{
MIXED NORM ESTIMATES FOR POTENTIAL OPERATORS RELATED TO THE RADON TRANSFORM
}

\author{
JAVIER DUOANDIKOETXEA ${ }^{\bowtie}$ and OSANE ORUETXEBARRIA
}

(Received 25 May 2005; accepted 22 August 2008)

Communicated by J. J. Koliha

\begin{abstract}
We define potential operators on hyperplanes and give sharp mixed norm inequalities for them. One of the operators coincides with the Radon transform for which mixed norm estimates are known but in reverse order. Those inequalities will be crucial in our proofs.
\end{abstract}

2000 Mathematics subject classification: 42B25.

Keywords and phrases: potential operators, Radon transform, mixed norm estimates.

\section{Introduction}

The Radon transform of a smooth function is defined as its integral on hyperplanes. Hyperplanes can be described as $\{x:\langle x, u\rangle=t\}$ for fixed $u \in S^{n-1}$ and $t \in \mathbb{R}$; with this notation,

$$
R f(t, u)=\int_{\langle x, u\rangle=t} f(x) d x,
$$

defines the Radon transform of $f$ (the integral is with respect to the $(n-1)$ dimensional Lebesgue measure on the hyperplane). Note that each hyperplane is taken twice and $R f(t, u)=R f(-t,-u)$. The Radon transform is an operator with many mathematical and practical applications (see [4] and [8]).

In this paper we consider a family of operators that contains the Radon transform. More precisely, we define Riesz potential operators on each hyperplane and define for $0<\alpha \leq n$ the operator

$$
T_{\alpha} f(x, u)=\int_{u^{\perp}} f(x-y)|y|^{\alpha-n+1} d y .
$$

Both authors supported in part by grant BFM2002-01550 of MCYT (Spain) and FEDER, and by European Project HPRN-CT-2001-00273-HARP.

(C) 2008 Australian Mathematical Society 1446-7887/08 \$A2.00+0.00 
Although ( $n-1)$-dimensional Riesz potentials are defined only for $0<\alpha \leq n-1$, we allow all values of $\alpha$ up to $n$ like in the $n$-dimensional potentials. Here $T_{n-1}$ is essentially the Radon transform with a different parameterization, namely,

$$
R f(\langle x, u\rangle, u)=T_{n-1} f(x, u) .
$$

Then $T_{n-1}$ depends on $\langle x, u\rangle$ rather than on $x$, but this is not the case for the other values of $\alpha$.

We are interested in mixed norm inequalities of the form

$$
\left(\int_{\mathbb{R}^{n}}\left(\int_{S^{n-1}}\left|T_{\alpha} f(x, u)\right|^{r} d \sigma(u)\right)^{q / r} d x\right)^{1 / q} \leq C_{p, q, r}\|f\|_{p} .
$$

In [7] we studied a similar problem for directional potential operators and in [6] we considered potential operators over $k$-planes but acting only on radial functions. The results in the last paper were sharp, while the results in [7] were sharp only for $\alpha>n /(n+1)$.

Apart from those appearing in [6], we could not find any mixed norm inequalities for $T_{\alpha}$ except for $\alpha=n-1$ (Radon transform) in the literature. However, even in that case, the inequalities in (1) are defined for the operator $R$ rather than for $T_{n-1}$. These are due to Oberlin and Stein [9], and are reproduced in Theorem 1.2. Two important differences with our estimates are that the mixed norm is taken in reversed order and that one of the integrals is on $\mathbb{R}$ and not on $\mathbb{R}^{n}$. For this second difference it is important to realize that an inequality like (3) with the norms in the left-hand side in reversed order would not be possible for $T_{n-1}$. Indeed, since for fixed $u, T_{n-1} f(x, u)$ depends only on $\langle x, u\rangle$,

$$
\int_{\mathbb{R}^{n}}\left|T_{n-1} f(x, u)\right|^{q} d x,
$$

becomes infinity. We consider some mixed norm inequalities in reversed order with the integral in $x$ on all of $\mathbb{R}^{n}$ in Section 3.2. They will appear by applying Minkowski's integral inequality to the left-hand side of (3) and hold only for some values of $\alpha$.

The main theorem we prove in this paper is the following.

THEOREM 1.1. Let $n \geq 3$. The condition $1 / p-1 / q=\alpha / n$ is necessary for (3) to hold. Assuming that it is satisfied, we have the following results.

(i) Let $n-1 \leq \alpha \leq n$. Here $T_{\alpha}$ satisfies inequality (3) if and only if

$$
\frac{1}{r}>\frac{n}{p}-(n-1), \quad \frac{1}{r} \geq \frac{(n-1) \alpha}{n}-n+2 .
$$

(ii) Let $(n(n-1)) /(n+1) \leq \alpha \leq n-1$. Here $T_{\alpha}$ satisfies inequality (3) if and only if

$$
\frac{1}{r}>\frac{n}{p}-(n-1), \quad \frac{1}{r} \geq \frac{\alpha}{n(n-1)} .
$$




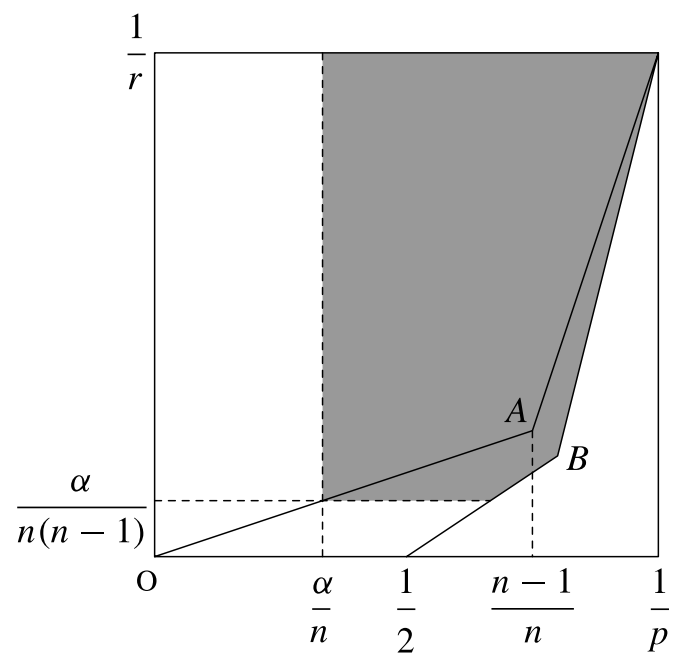

FIGURE 1. Case $0<\alpha<(n(n-1)) /(n+1)$.

(iii) Let $0<\alpha<(n(n-1)) /(n+1)$. Here $T_{\alpha}$ satisfies inequality (3) if

$$
\frac{1}{r}>\frac{n}{p}-(n-1), \quad \frac{n-1}{r}>\frac{2}{p}-1, \quad \frac{1}{r}>\frac{\alpha}{n(n-1)},
$$

and if $r=n(n-1) / \alpha$ and $(n-1) / \alpha \leq p<n / \alpha$. It does not for other values of $p$ and $r$ except maybe when $r=n(n-1) / \alpha$ and $2 n /(n+\alpha)<p<(n-1) / \alpha$.

The theorem is almost sharp; there is only a small undecided segment in part (iii). Like the usual $n$-dimensional potential operators there is a weaker result at the endpoint $p=n / \alpha(q=\infty)$ which we consider in Section 4 of the paper.

Figures 1 and 2 show the boundedness region in two different situations. Write $A$, $B, C$ and $O$ and $I$ for $((n-1) / n, 1 / n),(n /(n+1), 1 /(n+1)),(1 / 2,0),(0,0)$ and $(1,1)$. The lower left corner lies on the line $O A$, or on the line $A I$, and the lower right corner lies on the line $B C$ or on the line $B I$; these two lines are the right-hand edges of the boundedness region. Since the left vertical side is contained in the line $1 / p=\alpha / n$, this is enough to complete the picture of the boundedness polygon.

The boundedness region increases as $\alpha$ decreases and the union of those regions for $\alpha>0$ is exactly the boundedness region obtained for the mixed norm inequalities for the maximal function over hyperplanes. This result appears in [3] and is reproduced as Theorem 3.1 here because it is useful in the proof of the theorem.

Mixed norm estimates for the Radon transform defined in (1) were obtained by Oberlin and Stein, as we mentioned above. The theorem proved in [9] is as follows. 


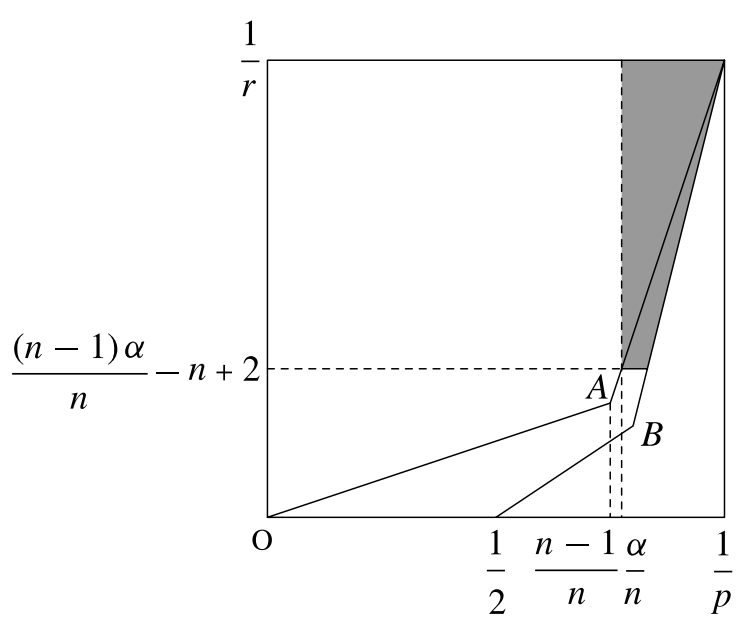

FiguRE 2. Case $n-1<\alpha \leq n$.

THEOREM 1.2. Let $n \geq 3$. The inequality

$$
\left(\int_{S^{n-1}}\left(\int_{-\infty}^{\infty}|R f(t, u)|^{q} d t\right)^{r / q} d \sigma(u)\right)^{1 / r} \leq C_{p, q, r}\|f\|_{p},
$$

holds if and only if

$$
1 \leq p<\frac{n}{n-1}, \quad r \leq p^{\prime} \quad \text { and } \quad \frac{1}{q}=\frac{n}{p}-(n-1) .
$$

The inequality also holds when $f$ is the characteristic functions of a measurable set if $p=n /(n-1)$ (equivalently, it holds in the Lorentz space $\left.L^{n /(n-1), 1}\right)$.

We use the Lorentz space $L^{p, 1}$ several times. For the definition and properties of Lorentz spaces, including the interpolation theorems, we refer the reader to [10, Chapter V]. For the interpolation results between vector-valued $L^{p}$ spaces used in Section 3 we refer the reader to [1, Chapter 5].

\section{Necessary conditions}

(1) The condition $1 / p-1 / q=\alpha / n$ is obtained by homogeneity: change $f(x)$ into $f(\lambda x)$ and require the constant of the inequality to be independent of $\lambda$. Since $1 / q$ cannot be negative, $p$ must not be greater than $n / \alpha$. More precisely, the function

$$
f(x)=(2+|x|)^{-n / p}(\log (2+|x|))^{-1},
$$

shows that the inequality is not possible even for $p=n / \alpha$. Nevertheless, we shall see later that it holds if $L^{n / \alpha}$ is replaced with the smaller Lorentz space $L^{n / \alpha, 1}$. 
(2) Taking as $f$ the characteristic function of a ball and requiring integrability at the left-hand side of (3), the following condition is obtained:

$$
\frac{1}{r}>\frac{n}{p}-(n-1) \text {. }
$$

(3) Let

$$
E=\left\{x: x_{1}^{2}+\cdots+x_{n-1}^{2} \leq \epsilon^{2}, x_{n} \in[-1 / 2,1 / 2]\right\}
$$

and

$$
A=\left\{x: x_{1}^{2}+\cdots+x_{n-1}^{2} \leq(\epsilon / 2)^{2}, x_{n} \geq 2\right\} .
$$

We proceed by taking as $f$ the characteristic function of $E$ to obtain $T_{\alpha} \chi_{E}(x, u) \sim C \epsilon^{n-2}|x|^{\alpha-n+1}$ for $x \in A$ when the direction $u$ moves into a set of measure of the order $\epsilon /|x|$. Then the left-hand side of (3) is finite only if (6) holds. Moreover, if we compare the exponents of $\epsilon$ on both sides of (3) and let $\epsilon$ tend to zero, the following condition is obtained:

$$
\frac{1}{r} \geq \frac{(n-1) \alpha}{n}-n+2 .
$$

This condition is meaningful only when $\alpha \geq(n-2) n /(n-1)$, since $r$ must be positive.

(4) Let

$$
E=\left\{x: x_{1}^{2}+x_{2}^{2} \leq \epsilon^{2},-1 / 2 \leq x_{3}, \ldots, x_{n} \leq 1 / 2\right\}
$$

and

$$
A=\left\{x: x_{1}^{2}+x_{2}^{2} \geq 4,-1 / 2 \leq x_{3}, \ldots, x_{n} \leq 1 / 2\right\}
$$

( $A$ is independent of $\epsilon$ ). Taking as $f$ the characteristic function of $E$ we have $T_{\alpha} \chi_{E}(x, u) \sim C \epsilon|x|^{\alpha-n+1}$ for $x \in A$ when the direction $u$ moves into a set of measure of the order $(\epsilon /|x|)^{n-1}$. The left-hand side of (3) is finite when (6) holds. On the other hand, comparing the exponents of $\epsilon$ on both sides of (3) and letting $\epsilon$ tend to zero, the following condition appears:

$$
\frac{n-1}{r}>\frac{2}{p}-1 \text {. }
$$

(5) Finally, let

$$
E=\left\{x: x_{1}^{2}+\cdots+x_{n-1}^{2} \leq 1,\left|x_{n}\right| \leq \epsilon\right\}
$$

and

$$
A=\left\{x: x_{1}^{2}+\cdots+x_{n-1}^{2}>4,\left|x_{n}\right|<\epsilon\right\} .
$$

Taking as $f$ the characteristic function of $E$ we have $T_{\alpha} \chi_{E}(x, u) \sim C|x|^{\alpha-n+1}$ for $x \in A$ when the direction $u$ moves into a set of measure of the order $(\epsilon /|x|)^{n-1}$. Again the left-hand side of (3) is finite if (6) holds, and comparing the exponents of $\epsilon$ the following condition is deduced:

$$
\frac{1}{r} \geq \frac{\alpha}{n(n-1)} \text {. }
$$




\section{Proof of Theorem 1.1 (Sufficiency)}

Define the Hardy-Littlewood maximal operator on hyperplanes as

$$
M f(x, u)=\sup _{R>0} \frac{1}{R^{n-1}} \int_{\left\{y \in u^{\perp}:|y|<R\right\}}|f(x-y)| d y .
$$

For fixed $u$ this is a bounded operator on $L^{p}\left(\mathbb{R}^{n}\right)$ with bounds independent of $u$ as a consequence of the boundedness of the usual Hardy-Littlewood maximal operator and Fubini's theorem. The mixed norm inequalities of the type (3) for $M$ are given by the following theorem.

THEOREM 3.1 (Christ et al. [3]). For $n \geq 3$, the operator $M f(x, u)$ is bounded from $L^{p}$ to $L^{p}\left(L^{r}\right)$ if and only if

$$
1 \leq r<\infty, \quad \frac{1}{r}>\frac{n}{p}-(n-1) \quad \text { and } \quad \frac{n-1}{r}>\frac{2}{p}-1 .
$$

We need this theorem and the following lemma in the proof of Theorem 1.1.

LEMMA 3.2. The following pointwise inequalities hold for nonnegative $f$.

(i) Let $0<\beta<\alpha<\gamma \leq n$. Then

$$
T_{\alpha} f(x, u) \leq T_{\beta} f(x, u)^{1-s} T_{\gamma} f(x, u)^{s}, \quad \alpha=(1-s) \beta+s \gamma .
$$

(ii) Let $0<\alpha<\gamma \leq n$. Then there exists a constant $C$ depending only on $\alpha, \gamma$ and $n$ such that

$$
T_{\alpha} f(x, u) \leq C M f(x, u)^{1-\alpha / \gamma} T_{\gamma} f(x, u)^{\alpha / \gamma} .
$$

The first part of the lemma is a consequence of Hölder's inequality. The second part is an inequality of Hedberg type and can be proved decomposing the integral into two parts and choosing them conveniently (see a proof in [6]).

Comparing both parts of the lemma we can say that the maximal operator $M$ takes the place of the operator $T_{\alpha}$ corresponding to $\alpha=0$.

It is interesting to compare the present situation with that of [7] for directional operators. There we did not know the optimal result for the associated maximal operator; if it were known, then mixed norm inequalities for directional potential operators would be a direct consequence of the analogue of Lemma 3.2 [7, Lemma 3.3]. Here the boundedness regions for different values of $\alpha$ are not homothetic to each other and Theorem 1.1 is not a corollary of Theorem 3.1. Two critical values of $\alpha$ appear, namely, $\alpha=n(n-1) /(n+1)$ and $\alpha=n-1$, corresponding to the values of $\alpha$ for which the points $A$ and $B$ of Figures 1 and 2 are vertices of the boundedness polygon. The strategy of the proof is to obtain the results for those critical values and use Lemma 3.2 and interpolation to complete the theorem. Since the operators are nonnegative, we assume from now on that $f$ is nonnegative. 
3.1. Case $r=1$ The equality

$$
\int_{S^{n-1}} \int_{u^{\perp}} g(y)|y| d y d \sigma(u)=\left|S^{n-2}\right| \int_{\mathbb{R}^{n}} g(y) d y,
$$

(see [8, p. 190]) applied to $g(y)=f(x-y)|y|^{\alpha-n}$ gives

$$
\int_{S^{n-1}} T_{\alpha} f(x, u) d \sigma(u)=C_{n} I_{\alpha} f(x),
$$

where $I_{\alpha}$ is (a multiple of) the Riesz potential of order $\alpha$ in $\mathbb{R}^{n}$. It is well known that $I_{\alpha}$ is bounded from $L^{p}$ to $L^{q}$ with $1 / q=1 / p-\alpha / n$ and $1<p<n / \alpha$. This gives the upper side of the boundedness polygon in Theorem 1.1 for all $\alpha$.

3.2. Case $\alpha=n(n-1) /(n+1)$ and $r=n+1$ Let $0<\alpha<n-1$. Here $T_{\alpha}$ is an $(n-1)$-dimensional fractional integral (Riesz potential) on the hyperplane $u^{\perp}$. This operator is bounded from $L^{p_{0}}\left(\mathbb{R}^{n-1}\right)$ to $L^{q_{0}}\left(\mathbb{R}^{n-1}\right)$ with $1 / q_{0}=1 / p_{0}-\alpha /(n-1)$.

If $q \geq r$ we can apply Minkowski's integral inequality to write

$$
\begin{aligned}
& \left(\int_{\mathbb{R}^{n}}\left(\int_{S^{n-1}} T_{\alpha} f(x, u)^{r} d \sigma(u)\right)^{q / r} d x\right)^{r / q} \\
& \quad \leq \int_{S^{n-1}}\left(\int_{\mathbb{R}^{n}} T_{\alpha} f(x, u)^{q} d x\right)^{r / q} d \sigma(u) .
\end{aligned}
$$

The inner integral on $\mathbb{R}^{n}$ splits into an integral on the direction $u$ and an $(n-1)$ dimensional integral on $u^{\perp}$. Let $p_{0}$ be defined by $1 / p_{0}=1 / q+\alpha /(n-1)$. Using the boundedness of the $(n-1)$-dimensional fractional integral on $u^{\perp}$ the right-hand side of (8) is bounded by

$$
C \int_{S^{n-1}}\left(\int_{-\infty}^{+\infty}\left(R f^{p_{0}}(t, u)\right)^{q / p_{0}} d t\right)^{r / q} d \sigma(u)
$$

where $R$ is the Radon transform defined in (1). Now using the estimate (5) under the conditions of Theorem 1.2, this is bounded by $C\left\|f^{p_{0}}\right\|_{p_{1}}^{r / p_{0}}$ whenever

$$
q_{1}=\frac{q}{p_{0}}, \quad r_{1}=\frac{r}{p_{0}}, \quad p_{1}=\frac{p}{p_{0}}, \quad 1 \leq p_{1}<\frac{n}{n-1}, \quad r_{1} \leq p_{1}^{\prime} .
$$

(The relation between $p_{1}$ and $q_{1}$ required in the theorem is a consequence of the relation between $p$ and $q$, and the definition of $p_{0}$.) Then we have proved (3) for

$$
0<\alpha<n-1, \quad \frac{\alpha}{n}<\frac{1}{p} \leq \frac{1}{r}+\frac{\alpha}{n}, \quad \frac{1}{p}+\frac{\alpha}{n(n-1)}<1 .
$$

The last conditions are due to $q \geq r$ and $p_{0}>1$.

In the particular case $\alpha=n(n-1) /(n+1), r=n+1$, the range we obtain fits exactly with the (open) lower side of the boundedness polygon. 
3.3. Case $\alpha=n-1$ and $\boldsymbol{r}=\boldsymbol{n}$ We now prove the estimate corresponding to $\alpha=n-1, r=n$, and $n^{2} /\left(n^{2}-n+1\right)<p<n /(n-1)$ (that is, $\left.n^{2}<q<\infty\right)$.

The adjoint operator to the Radon transform $R$ defined in (1) is

$$
R^{*} g(x)=\int_{S^{n-1}} g(\langle x, u\rangle, u) d \sigma(u) .
$$

The mixed norm estimates satisfied by $R^{*}$ are obtained dualizing those in Theorem 1.2. Using (2) we have

$$
\int_{S^{n-1}} T_{n-1} f(x, u)^{n} d \sigma(u)=R^{*}\left((R f)^{n}\right)(x) .
$$

Then

$$
\begin{aligned}
\left\|T_{n-1} f\right\|_{L^{q}\left(L^{n}\right)}^{n} & =\left\|R^{*}\left((R f)^{n}\right)\right\|_{q / n} \leq C\left\|(R f)^{n}\right\|_{L^{q / n^{2}}\left(L^{q /(q-n)}\right)} \\
& =C\|R f\|_{L^{q / n}\left(L^{n q /(q-n)}\right)}^{n} \leq C\|f\|_{p}^{n},
\end{aligned}
$$

which is the desired estimate.

\subsection{How to complete the boundedness regions}

(a) Let $\alpha=n-1$. Interpolating the result for $r=n$ obtained above with the result for $r=1$, we obtain the result for $1<r<n$.

For $n-1<\alpha<n$, it is enough to prove the theorem for the largest possible value of $r$, that is, $r_{c}=n /\left[(n-1) \alpha-n^{2}+2 n\right]$ and interpolate with $r=1$. We use the first part of Lemma 3.2 with $\beta=n-1, \gamma=n, s=\alpha-n+1$ and Hölder's inequality to obtain

$$
\begin{aligned}
\int_{S^{n-1}} T_{\alpha} f(x, u)^{r_{c}} d \sigma(u) \leq & \left(\int_{S^{n-1}} T_{n-1} f(x, u)^{\left((1-s) r_{c}\right) /\left(1-s r_{c}\right)} d \sigma(u)\right)^{1-s r_{c}} \\
& \times\left(\int_{S^{n-1}} T_{n} f(x, u) d \sigma(u)\right)^{s r_{c}},
\end{aligned}
$$

and the last integral is a multiple of $\|f\|_{1}$ according to (7). Since the exponent $(1-s) r_{c} /\left(1-s r_{c}\right)$ is equal to $n$, we use the boundedness of $T_{n-1}$ from $L^{p_{0}}$ to $L^{(1-s) q}\left(L^{n}\right)$ (for the appropriate value of $p_{0}$ ) obtained in the preceding subsection and eventually obtain

$$
\left(\int_{\mathbb{R}^{n}}\left(\int_{S^{n-1}} T_{\alpha} f(x, u)^{r_{c}} d \sigma(u)\right)^{q / r_{c}} d x\right)^{1 / q} \leq C\|f\|_{p_{0}}^{1-s}\|f\|_{1}^{s} .
$$

When $f$ is the characteristic function of a set $E$, the right-hand side is $|E|^{1 / p}$, which is the desired result but only for such characteristic functions, or for the Lorentz space $L^{p, 1}$. Moreover, we can check that all of the values of $p$ allowed in Theorem 1.1 for $r_{c}$ are obtained. Fixing two such values of $p$, interpolation between Lorentz spaces gives the result in the intermediate Lorentz spaces; since the range of values of $p$ is open we obtain the desired result. 
(b) Let $\alpha=n(n-1) /(n+1)$. Interpolate the result of Section 3.2 above with the result for $r=1$.

For $n(n-1) /(n+1)<\alpha<n-1$ we proceed as in the previous case (a) and obtain the line corresponding to the largest value of $r$ : we now use the first part of Lemma 3.2 with $\beta=n(n-1) /(n+1)$ and $\gamma=n-1$, and the results for $T_{n(n-1) /(n+1)}$ and $T_{n-1}$, then specialize to characteristic functions of sets and finally interpolate.

(c) Let $\alpha<n(n-1) /(n+1)$ and $n+1<r<n(n-1) / \alpha$. Use the second part of Lemma 3.2 with $\gamma=n(n-1) /(n+1)$ and Hölder's inequality to obtain

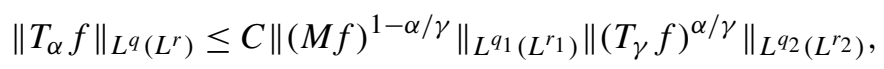

with $1 / q_{1}+1 / q_{2}=1 / q$ and $1 / r_{1}+1 / r_{2}=1 / r$. Choose $r_{2}=n(n-1) / \alpha$ and apply the result of Section 3.2 to $T_{\gamma}$ and Theorem 3.1 to $M$. Two different norms of $f$ appear again and we go through characteristic functions and interpolation in Lorentz spaces to obtain strong estimates. For $r<n(n-1) / \alpha$ it suffices to interpolate with the result for $r=1$.

Note that we have proved the result only for the open range $r<n(n-1) / \alpha$. When $r=n(n-1) / \alpha$ we know the boundedness for $(n-1) / \alpha \leq p<n / \alpha$ from Section 3.2, and it seems reasonable to expect that the boundedness holds also for $2 n /(n+\alpha)<p<(n-1) / \alpha$.

\section{The estimate for $p=n / \alpha$}

The estimate (3) corresponding to $p=n / \alpha$ and $q=\infty$ is false but it holds for the range of values of $r$ allowed in Theorem 1.1 if $L^{n / \alpha}$ is replaced with the smaller Lorentz space $L^{n / \alpha, 1}$. It suffices to prove this for $\alpha=n-1$ and use Lemma 3.2 for other values of $\alpha$.

Let $\alpha=n-1, r=n, p=n /(n-1)$ and $q=\infty$. Using the translation invariance of the integral defining $T_{n-1}$ we need to see that

$$
\left(\int_{S^{n-1}}\left(\int_{u^{\perp}} f(y) d y\right)^{n} d \sigma(u)\right)^{1 / n} \leq C\|f\|_{n /(n-1), 1} .
$$

When $f$ is the characteristic function of a measurable set $E$ this has the following geometrical interpretation: consider the $(n-1)$-dimensional measures of the sections of $E$ with all of the hyperplanes through a fixed point; the average on $S^{n-1}$ of the $n$th powers of those measures is not larger than the $(n-1)$ th power of the measure of $E$. Note that when $E$ is a ball of radius $R$ and the fixed point is the center of the ball, the measure of the sections is $c_{n} R^{n-1}$ and the measure of $E$ is $C_{n} R^{n}$, and the exponents match exactly.

Inequality (9) is weaker than the endpoint result of Theorem 1.2 corresponding to $p=n /(n-1)$. Indeed, in that case we had

$$
\left(\int_{S^{n-1}}\left(\sup _{t} \int_{\langle y, u\rangle=t} f(y) d y\right)^{n} d \sigma(u)\right)^{1 / n} \leq C\|f\|_{n /(n-1), 1} .
$$


(This says that in the above geometrical interpretation we can replace the section produced by a hyperplane with the largest of the sections produced by its translates.)

For a direct proof of (9) without using Theorem 1.2 we can proceed as follows. The left-hand side of (9) coincides with

$$
\sup \left\{\int_{S^{n-1}} \int_{u^{\perp}} f(y) d y g(u) d \sigma(u): g \in L^{n /(n-1)}\left(S^{n-1}\right) \text { with norm } 1\right\} .
$$

Using Fubini's theorem the double integral coincides with

$$
\int_{\mathbb{R}^{n}} f(y) G(y) d y,
$$

where

$$
G(y) d y=\int_{S^{n-1}} g(u) d \mu_{u}(y) d \sigma(u),
$$

and $d \mu_{u}$ is the tensor product of the $(n-1)$-dimensional Lebesgue measure on the hyperplane $u^{\perp}$ and the one-dimensional Dirac delta on the line $\{t u: t \in \mathbb{R}\}$. Using the duality between $L^{n /(n-1), 1}$ and $L^{n, \infty}$ we need to prove that $G$ is in $L^{n, \infty}$ with norm bounded by the $L^{n /(n-1)}$-norm of $g$ over $S^{n-1}$. Since $G$ is homogeneous of degree -1 , its norm in $L^{n, \infty}\left(\mathbb{R}^{n}\right)$ is (a multiple) of the $L^{n}$-norm of its restriction to the unit sphere. Then the problem is reduced to the boundedness of the operator

$$
g \mapsto G(v)=\int_{\langle u, v\rangle=0} g(u) d \sigma_{v^{\perp}}(u),
$$

from $L^{n /(n-1)}\left(S^{n-1}\right)$ to $L^{n}\left(S^{n-1}\right)$. This result is [2, Theorem 2.1(B)] (see also [5, Theorem 1]).

\section{Acknowledgements}

The authors are grateful to Adela Moyua and Edurne Seijo for interesting discussions about the subject of the paper.

\section{References}

[1] J. Bergh and J. Löfström, Interpolation Spaces. An Introduction (Springer, Berlin, 1976).

[2] F. M. Christ, 'Estimates for the $k$-plane transform', Indiana Univ. Math. J. 33 (1984), 891-910.

[3] M. Christ, J. Duoandikoetxea and J. L. Rubio de Francia, 'Maximal operators related to the Radon transform and the Calderón-Zygmund method of rotations', Duke Math. J. 53 (1986), 189-209.

[4] S. R. Deans, The Radon Transform and Some of Its Applications (John Wiley \& Sons, New York, 1983).

[5] S. Drury, 'A survey of k-plane estimates', Contemp. Math. 91 (1989), 43-55.

[6] J. Duoandikoetxea, V. Naibo and O. Oruetxebarria, ' $k$-plane transforms and related operators on radial functions', Michigan Math. J. 49 (2001), 265-276.

[7] J. Duoandikoetxea and O. Oruetxebarria, 'Mixed norm inequalities for directional operators associated to potentials', Potential Anal. 15 (2001), 273-283. 
[8] F. Natterer, The Mathematics of Computerized Tomography (B. G. Teubner, Stuttgart/John Wiley \& Sons, Chichester, 1986).

[9] D. M. Oberlin and E. M. Stein, 'Mapping properties of the Radon transform', Indiana Univ. Math. J. 31 (1982), 641-650.

[10] E. M. Stein and G. Weiss, Introduction to Fourier Analysis in Euclidean Spaces (Princeton University Press, Princeton, NJ, 1971).

JAVIER DUOANDIKOETXEA, Departamento de Matemáticas, Universidad del País Vasco-Euskal, Herriko Unibertsitatea, Apartado 644, 48080 Bilbao, Spain

e-mail: javier.duoandikoetxea@ehu.es

OSANE ORUETXEBARRIA, Departamento de Matemáticas,

Universidad del País Vasco-Euskal, Herriko Unibertsitatea, Apartado 644, 48080 Bilbao, Spain

e-mail: osane.oruetxebarria@ehu.es 